(2) Open Access Full Text Article

\title{
Assessment of hydroxychloroquine maculopathy after cessation of treatment: an optical coherence tomography and multifocal electroretinography study
}

This article was published in the following Dove Press journal:

Drug Design, Development and Therapy

II June 2015

Number of times this article has been viewed

\author{
Marilita M Moschos' \\ Eirini Nitoda' \\ Irini $P$ Chatziralli' \\ Zisis Gatzioufas ${ }^{2}$ \\ Chryssanthi Koutsandrea' \\ George Kitsos ${ }^{3}$ \\ 'First Department of Ophthalmology, \\ Medical School, National \\ and Kapodistrian University \\ of Athens, Athens, Greece; ${ }^{2}$ Swiss \\ Eye Research Foundation, Orasis \\ Eye Clinic, Reinach, Switzerland; \\ ${ }^{3}$ Department of Ophthalmology, \\ Medical School, University \\ of Ioannina, loannina, Greece
}

Objective: This study was conducted to evaluate the macular status of patients treated with hydroxychloroquine before and after cessation of treatment.

Methods: Forty-two patients with systemic lupus erythematosus underwent ocular examination based on visual acuity evaluation, optical coherence tomography retinal thickness measurements, and multifocal electroretinography (mfERG) records at first visit. The tests were repeated 6 months after treatment withdrawal and compared to the findings at their first visit.

Results: Mean visual acuity (measured in log minimum angle of resolution) of both eyes was statistically increased after hydroxychloroquine discontinuation (difference in means: 0.06 $[P<0.0001]$ and $0.01[P=0.003]$ for the right and left eyes, respectively). Retinal response amplitudes of central and peripheral areas were significantly improved for both eyes. The following values were observed for central responses: the difference in means was $-19.9(P<0.0001)$ and $-13.6(P<0.0001)$ for the right eye and the left eye, respectively; for peripheral responses, difference in means was $-10.3(P<0.0001)$ and $-9.5(P<0.0001)$ for right eye and left eye, respectively, after the 6-month examination. There were no statistically significant differences in the retinal thickness of patients after cessation of treatment. The visual acuity of the patients was correlated to central and peripheral mfERG responses $(r=-0.53[P<0.0001]$ and $r=-0.53$ $[P<0.0001]$, for the right eye and the left eye, respectively).

Conclusion: The visual acuity of patients receiving hydroxychloroquine improves along with the amplitudes of the mfERG responses 6 months after discontinuation of the drug, but no difference in retinal thickness is identified.

Keywords: hydroxychloroquine, toxic retinopathy, visual acuity, multifocal electroretinography, optical coherence tomography

\section{Introduction}

Chloroquine and hydroxychloroquine are the most common antimalarials used for the treatment of autoimmune and connective tissue diseases, including systemic lupus erythematosus, rheumatoid arthritis, psoriatic arthritis, palindromic rheumatism, dermatomyositis, Sjögren's syndrome, and juvenile chronic arthritis. ${ }^{1}$ Although chloroquine and hydroxychloroquine are chemically similar 4-aminoquinolines, differing only by an ethyl group, the latter exhibits almost half the efficacy and toxicity as compared with the former. ${ }^{1}$ Modification of the cellular microenvironment induces changes in inflammatory mediators and receptor activity, as well as changes in production of proteins and autoantibodies. ${ }^{1}$ The most common side effects of antimalarials include gastrointestinal cramps and nausea, skin lesions, and central nervous system symptoms that are usually reversible. ${ }^{1}$ Hydroxychloroquine has been also implicated in cardiomyopathy, vacuolar myopathy, and hypoglycemia observed in patients with type 2 diabetes mellitus, while massive
Correspondence: Marilita M Moschos First Department of Ophthalmology, Medical School, National and Kapodistrian University of Athens, 154 Mesogion Street, Athens II527, Greece Email moschosmarilita@yahoo.fr 
liver cell necrosis has been also referred to as a side effect of hydroxychloroquine. ${ }^{2-6}$ Hyperpigmented deposits of nails, mucosa, and skin, composed of melanin and hemosiderin within histiocytes, are frequently seen 1 year after therapy, while they start fading 2-6 months after cessation of the drug. ${ }^{7}$

Deposits of antimalarials on cornea, ciliary body, lens, and retina have been associated with ocular side effects, usually reversible after the discontinuation of treatment. ${ }^{8}$ Antimalarial drug-induced keratopathy patterns vary from diffuse punctuate opacities to radial and whirling lines (Hudson-Stahli line), resulting in halos and photophobia. ${ }^{8}$ Although defective accommodation and lens opacities have been described with chloroquine, these are rare complications under hydroxychloroquine treatment. ${ }^{8}$ The prevalence of hydroxychloroquine retinopathy ranges from $0 \%$ to $4 \%$ and it is related to the daily dose, the duration of treatment, the presence of other retinal disease, as well as the kidney and liver function. ${ }^{9}$ However, some authors estimate that the percentage of toxic maculopathy reaches up to $13.1 \%{ }^{10}$ The risk of hydroxychloroquine-induced toxicity has been appreciated to be of very low risk for doses $<6.5 \mathrm{mg} / \mathrm{kg} /$ day (200-400 mg/day) and a cumulative dose $<1,000$ g. ${ }^{8,9,11}$ The toxicity of the drug is based on disturbances in ganglion cells and photoreceptors, in addition to the binding of melanin to the retinal pigment epithelium. ${ }^{9,11}$ Basic ocular examination is recommended before starting treatment and then, annual examination, including visual acuity measurement, fundoscopy, and visual field examination, should be done. ${ }^{9,11}$ Fundoscopy is normal in early stages, followed by pigment disturbances, vascular attenuation, and finally, bull's eye maculopathy in advanced stages. ${ }^{8,9}$ Visual field examination reveals subtle usually superior scotomas, within ten grades of fixation, which are enlarged over time. ${ }^{9}$ Retinal dysfunction even in early stages can be defined with multifocal electroretinography (mfERG), while spectral domain optical coherence tomography (spectral domain-OCT) can detect thinning of retinal layers. . $^{9,11,12}$ The follow-up of the patients is essential, given that delayed onset of hydroxychloroquine retinopathy, 1 year after discontinuation of treatment, has also been reported..$^{8,13}$ The purpose of this study was to identify structural and functional changes in the retina of patients who stopped hydroxychloroquine treatment for 6 months, using optical coherence tomography and mfERG recording.

\section{Patients and methods}

\section{Patients}

Forty-two patients with systemic lupus erythematosus were included in this retrospective, nonrandomized study, which was conducted at the First University Eye Clinic of General Hospital of Athens G. Gennimatas. All patients received $400 \mathrm{mg} /$ day hydroyxchloroquine (tb Plaquenil $200 \mathrm{mg}$; Sanofi-Aventis, Laval, QC, Canada) in divided doses and the mean duration of the treatment was 12 months. After the first ocular examination all patients stopped hydroyxchloroquine according to their physician's advice. The cessation of treatment had been already decided and scheduled for all patients independently of our protocol. No toxicity was mentioned by patients' physicians. To calculate adjusted daily dose, the lesser of the ideal body weight based on height and actual body weight was used as the operative weight and the formula was daily dose (in $\mathrm{mg} /$ day)/[operative weight (in pounds) $/(2.2$ pounds $/ \mathrm{kg}$ )]. Three patients were found with dosages higher than the recommended $6.5 \mathrm{mg} / \mathrm{kg} /$ day and were excluded from our study. Patients included in the study had a cumulative dose of lower than 1,000 g. The protocol used was approved by the ethics committee of the University Hospital, and all patients gave written informed consent.

\section{Assessment of visual acuity}

Best-corrected visual acuity was measured for both eyes of all patients using Snellen optotype at the first visit and 6 months after the cessation of therapy.

\section{OCT examination}

OCT examination was performed with the Stratus OCT Model 3000 (Carl Zeiss Meditec, Dublin, CA, USA), using the retinal mapping software. All eyes were scanned in a radial spoke pattern centered on the foveola with scan length of $6 \mathrm{~mm}$.

The retinal thickness was calculated as the distance between the two boundaries along each A-scan, based on the attached automatic boundary detection software. The participants underwent the examination at the first visit and 6 months after the discontinuation of hydroxychloroquine.

\section{Multifocal electroretinography}

The records of the mfERG correspond to the combined responses of photoreceptors, bipolar cells, and Muller cells. mfERG was conducted using VERIS III visual evoked response imaging system (Tomey, Nagoya, Japan). The stimulus matrix consisted of 61 pattern scaled segments displayed on a cathode ray tube color monitor (Sony, Tokyo, Japan), driven at a frame rate of $72 \mathrm{~Hz}$. Each hexagon was independently alternated between black and white at a rate of $72 \mathrm{~Hz}$. The radius of the stimulus array was approximately $20^{\circ}$ high and $25^{\circ}$ wide. The bandwidth of the amplifier was $10-300 \mathrm{~Hz}$ 
and the amplification was $\times 10,000$. The pupils of the patients were dilated using tropicamide $0.5 \%$ and phenylephrine $5 \%$, and all patients were optically corrected for near vision. A gold-foil electrode was inserted under the lower eyelid, a reference one was placed on the ipsilateral skin, posterior to the lateral orbital rim, and the ground electrode was attached to the earlobe. The duration of the data acquisition was $4 \mathrm{~min}-$ utes, divided into four sessions of 60 seconds. Patients' fixation was monitored using a fundus camera system, while the fellow eye was closed. The recording procedure was repeated whenever there were spurious potentials from eye blinks or whenever ocular movements were recorded.

The response density (amplitude per unit retinal area, in nanovolts per square degree or $\mathrm{nV} / \mathrm{deg}^{2}$ ) of each local response was estimated as the dot product between the normalized response template and each local response. The following anatomic areas were defined for evaluation of the retinal responses: ring 1 to the fovea $\left(0^{\circ}-2^{\circ}\right)$, ring 2 to the parafovea $\left(2^{\circ}-7^{\circ}\right)$, ring 3 to the perifovea $\left(7^{\circ}-13^{\circ}\right)$, ring 4 to the near periphery $\left(3^{\circ}-22^{\circ}\right)$, and ring 5 to the central part of the middle periphery $\left(22.0^{\circ}-30.5^{\circ}\right)$. The amplitude of each group was scaled to reflect the angular size of the stimulus hexagon that produces the response. The protocol followed the recommended guidelines of the International Society of Electrophysiology of Vision for basic mfERG. All the patients underwent the examination at the first visit and 6 months after the cessation of hydroxychloroquine.

\section{Statistical analysis}

The statistical software SPSS Statistics 22.0 was used for the analysis. The normality of the distribution of all variables was controlled with nonparametric Kolmogorov-Smirnov analysis. Assessing the functional changes in vision related to discontinuation of hydroxychloroquine, individual comparisons of visual acuity measurements were made between the two eyes, based on nonparametric Wilcoxon test. The toxic effect of hydroxychloroquine on retina was also evaluated using paired $t$-tests for the values of retinal thickness and mfERG responses. The correlation between visual acuity and $\mathrm{mfERG}$ responses was expressed using Pearson's correlation coefficient.

\section{Results \\ Demographics}

Forty-two patients with systemic lupus erythematosus participated in this study, including 18 males (43\%) and 24 females (57\%), with mean age of 47 years (standard deviation [SD]: 13 years). The mean duration of hydroxychloroquine medication was 12 (SD: 11) months.

\section{Measurements of visual acuity}

The mean visual acuity (VA in log minimum angle of resolution $[\log \mathrm{MAR}])$ at the time of first ocular examination was 0.15 (SD: 0.034) and 0.09 (SD: 0.051) for the right (VARE1) and left (VALE1) eye, respectively. The corresponding values for the right eye (VARE2) were 0.09 (SD: 0.064) and for the left one (VALE2) 0.08 (SD: 0.058) at the second visit (6 months later). The distribution of visual acuity measurements is shown in Figure 1. Assessing the visual acuity, there is statistically significant increase for the right (difference in means: 0.06, standard error [SE]: 0.011, $P<0.0001)$ and left (difference in means: 0.01 , SE: $0.003, P=0.003$ ) eye 6 months later.

\section{Retinal thickness}

The calculations performed after the OCT revealed that the mean retinal thickness (in micrometers) of the right (RTRE1) and left (RTLE1) eye was 171.0 (SD: 15.2) and 169.1 (SD: 12.1), respectively, at the first examination. Six months later, the corresponding values for the right (RTRE2) and left (RTLE2) eye were 171.1 (SD: 14.3) and 170.4 (SD: 12.2), respectively. The mean values of retinal thickness (in micrometers) at the first and second visits are presented in the boxplots of Figure 2. Comparing the mean values of retinal thickness between the first examination and the second one 6 months after discontinuation of hydroxychloroquine, no statically significant difference is revealed either for the right eye (difference in means: $-0.1, \mathrm{SE}: 0.6$, $P=0.906$ ) or for the left one (difference in means: $-1.2, \mathrm{SE}$ : $0.8, P=0.150)$.

\section{Records of mfERG}

The mean values of the amplitudes (nanovolts per square degree) and implicit times (milliseconds) of both eyes' central ( $\mathrm{mf} 1$ and $\mathrm{T} 1$, respectively) and peripheral (mf2 and T2,

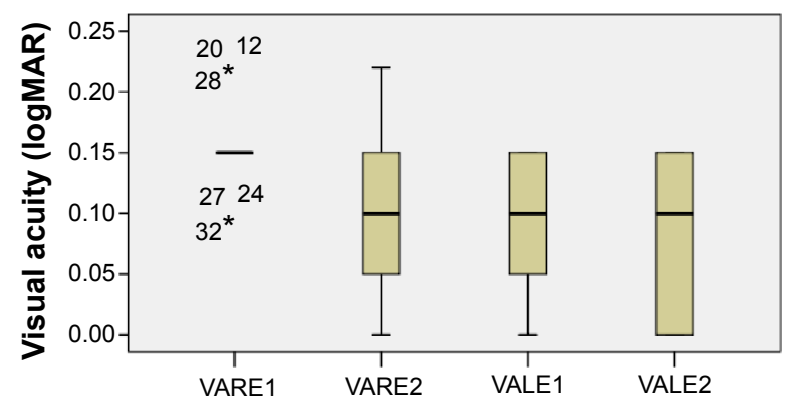

Figure I VA distribution.

Notes: Boxplots of VA (logMAR) mean values for each eye at the first and second visits.

Abbreviations: logMAR, log minimum angle of resolution; VA, visual acuity; VALEI and 2, VA of left eye at first and second visits, respectively; VAREI and 2, VA of right eye at first and second visits, respectively. 


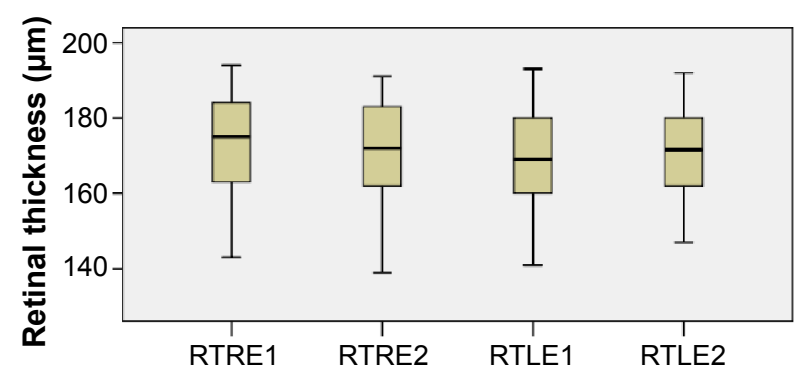

Figure 2 Retinal thickness distribution.

Notes: Boxplots of retinal thickness (in micrometers) mean values for each eye at the first and second visits.

Abbreviations: RTLEI and 2, retinal thickness of left eye at first and second visits, respectively; RTREI and 2, retinal thickness of right eye at first and second visits, respectively.

respectively) responses at the first and second (6 months later) visits are presented in Table 1 and Figures 3-6. There is a statistically significant increase in the central (right eye: difference in means -19.9 , SE: $3.4, P<0.0001$; left eye: difference in means $-13.6, \mathrm{SE}: 3.3, P<0.0001)$ and peripheral (right eye: difference in means $-10.3, \mathrm{SE}: 2.6, P<0.0001$; left eye: difference in means -9.5 , SE: $2.3, P<0.0001$ ) amplitudes of both eyes 6 months after discontinuation of hydroxychloroquine. There are no significant differences in the implicit times of central (right eye: difference in means $-0.1, \mathrm{SE}: 0.1$, $P=0.254$; left eye: difference in means 0.2 , SE: $0.1, P=0.037$ ) and peripheral (right eye: difference in means $-0.1, \mathrm{SE}: 0.2$, $P=0.650$; left eye: difference in means -0.2 , SE: $0.3, P=0.554$ ) responses of both eyes after the cessation of medication.

\section{Correlation of visual acuity with mfERG responses}

The visual acuity of the left eye seems to increase along with the amplitudes of central and peripheral responses $(r=-0.55$, $P<0.0001$ and $r=-0.46, P=0.002$, respectively) at the time of the first examination. However, there is no significant correlation between the aforesaid values assessing the right eye at the same time ( $r=0.09, P=0.586$ and $r=0.08, P=0.625$, respectively). A significant negative correlation is also detected between the patients' retinal responses and the visual acuity both at the central ( $r=-0.53, P<0.0001$ and $r=-0.53, P<0.0001$, for the right and left eyes, respectively) and at the peripheral ( $r=-0.45$, $P=0.003$ and $r=-0.62, P<0.0001$, for the right and left eyes, respectively) areas after 6 months without treatment. In addition, the visual acuity of both eyes seems to increase in parallel after the cessation of hydroxychloroquine $(r=0.85, P<0.0001)$, but there is no strong relation at the first examination $(r=0.02$, $P=0.915)$. Finally, no significant correlation is detected between the visual acuity of the right eye at the first and second visits ( $r=0.09, P=0.552$ ). However, the visual acuity values of the left eye before and after discontinuation of medication appear to be strongly correlated $(r=0.94, P<0.0001)$.

\section{Discussion}

Assessing hydroxychloroquine retinopathy, we presumed that there are statistically significant differences of visual acuity and amplitudes of mfERG responses between the first visit and after discontinuation of the drug 6 months later. Visual acuity (in $\log$ MAR) of patients participating in this study was increased for both eyes (right eye: difference in means 0.06, SE: 0.011, $P<0.0001$; left eye: difference in means $0.01, \mathrm{SE}: 0.003, P=0.003$ ) at the second visit. In addition, the amplitudes of the two eyes were significantly improved after treatment cessation for both central (right eye: difference in means -19.9 , SE: $3.4, P<0.0001$; left eye: difference in means -13.6 , SE: $3.3, P<0.0001)$ and peripheral (right eye: difference in means $-10.3, \mathrm{SE}: 2.6, P<0.0001$; left eye: difference in means -9.5 , SE: $2.3, P<0.0001)$ responses.

Table I Mean values and standard deviation of amplitudes $(\mathrm{mf})$ and implicit time $(\mathrm{T})$ of the central and peripheral responses at the first and second visits

\begin{tabular}{|c|c|c|c|c|}
\hline \multirow[b]{2}{*}{ Amplitudes (nV/deg $\left.{ }^{2}\right)$, mean (SD) } & \multicolumn{2}{|c|}{ First visit } & \multicolumn{2}{|c|}{ Second visit } \\
\hline & mfIREI & I54.7 (I7.2) & mfIRE2 & $174.5(19.5)$ \\
\hline & mfILEI & $163.6(16.2)$ & mfILE2 & I77.| (18.4) \\
\hline & mf2REI & $51.7(14.8)$ & mf2RE2 & $62.0(15.5)$ \\
\hline & mf2LEI & $57.1(15.0)$ & mf2LE2 & $66.6(17.1)$ \\
\hline \multirow[t]{4}{*}{ Implicit times (ms), mean (SD) } & TIREI & $41.1(0.7)$ & TIRE2 & $41.2(0.9)$ \\
\hline & TILEI & $4 I .5(0.9)$ & TILE2 & $4 I .3(0.8)$ \\
\hline & T2REI & $39.8(2.6)$ & T2RE2 & $39.9(2.2)$ \\
\hline & T2LEI & $40.1(1.7)$ & T2LE2 & $40.3(1.8)$ \\
\hline
\end{tabular}

Abbreviations: $\mathrm{mfIREI}$, amplitude, central area, right eye, first visit; mfILEI, amplitude, central area, left eye, first visit; mf2REI, amplitude, peripheral area, right eye, first visit; mf2LEI, amplitude, peripheral area, left eye, first visit; mfIRE2, amplitude, central area, right eye, second visit; mfILE2, amplitude, central area, left eye, second visit; mf2RE2, amplitude, peripheral area, right eye, second visit; mf2LE2, amplitude, peripheral area, left eye, second visit; TIREI, implicit time, central area, right eye, first visit; TILEI, implicit time, central area, left eye, first visit; T2REI, implicit time, peripheral area, right eye, first visit; T2LEI, implicit time, peripheral area, left eye, first visit; TIRE2, implicit time, central area, right eye, second visit; TILE2, implicit time, central area, left eye, second visit; T2RE2, implicit time, peripheral area, right eye, second visit; T2LE2, implicit time, peripheral area, left eye, second visit; nV/deg2, nanovolts per degree squared; SD, standard deviation. 


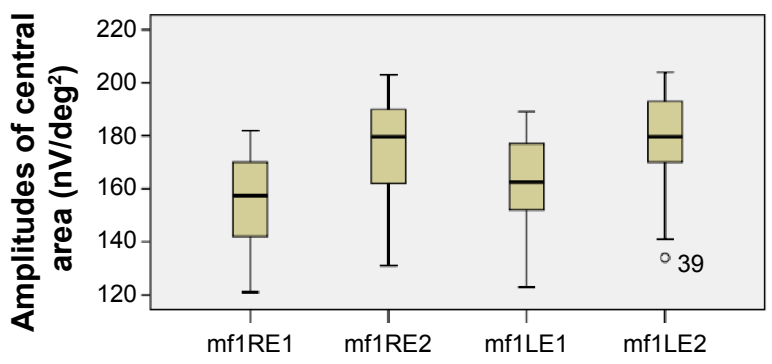

Figure 3 Amplitudes of central responses.

Notes: Boxplots of central amplitudes $\left(\mathrm{nV} / \mathrm{deg}^{2}\right)$ mean values for each eye at the first and second visits.

Abbreviations: LE, left eye; RE, right eye; mf, multifocal electroretinography reading; $\mathrm{mfIREI}$, central area, right eye, first visit; $\mathrm{mfIRE2}$, central area, right eye, second visit; $\mathrm{mfILEI}$, central area, left eye, first visit; mfILE2, central area, left eye, second visit; $\mathrm{nV} / \mathrm{deg}^{2}$, nanovolts per degree squared.

No statistically significant differences were observed in the implicit times of central and peripheral areas. A significant negative correlation was also detected between visual acuity and patients' retinal responses after discontinuation of hydroxychloroquine, while the visual acuity of both eyes appears to be improved in parallel at the same time. However, the retinal thickness of both eyes did not present any statistical difference between patients' examinations.

Abnormalities in mfERG have already been observed in patients taking hydroxychloroquine compared to healthy individuals. ${ }^{14}$ Central and pericentral depression was detected in patients' responses, even when the visual acuity was normal. ${ }^{14}$ The $\mathrm{mfERG}$ response is a sensitive functional index representing not only the retinal abnormalities induced by hydroxychloroquine but also the changes observed after treatment withdrawal. ${ }^{15}$ Improvement in mfERG response occurs along with visual acuity. ${ }^{15}$ Aliferis et $a{ }^{16}$ observed that the average implicit times of patients receiving hydroxychloroquine were significantly prolonged, but the amplitudes were

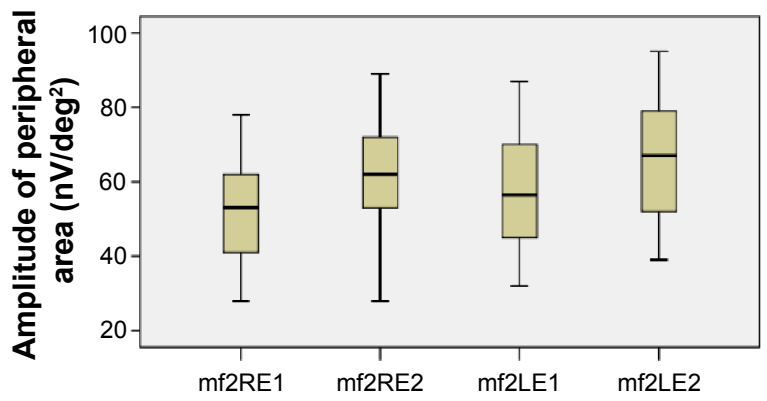

Figure 4 Amplitudes of peripheral responses.

Notes: Boxplots of peripheral amplitude $\left(\mathrm{nV} / \mathrm{deg}^{2}\right)$ mean values for each eye at the first and second visits.

Abbreviations: LE, left eye; RE, right eye; mf, multifocal electroretinography reading; $\mathrm{mf} 2 \mathrm{REI}$, peripheral area, right eye, first visit; $\mathrm{mf} 2 \mathrm{RE} 2$, peripheral area, right eye, second visit; mf2LEI, peripheral area, left eye, first visit; mf2LE2, peripheral area, left eye, second visit; $\mathrm{nV} / \mathrm{deg}^{2}$, nanovolts per degree squared.

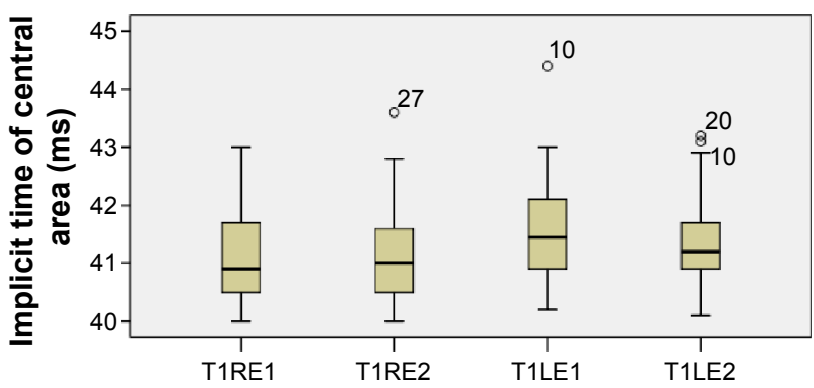

Figure 5 Implicit times of central responses.

Notes: Boxplots of central implicit time (ms) mean values for each eye at the first and second visits.

Abbreviations: LE, left eye; RE, right eye; TI, time I; TILEI, central area, left eye, first visit; TILE2, central area, left eye, second visit; TIREI, central area, right eye, first visit; TIRE2, central area, right eye, second visit.

not significantly impaired. They presumed that these values do not always change in parallel in toxic maculopathy caused by hydroxychloroquine. ${ }^{16}$ Melles and Marmor ${ }^{17}$ have recently reviewed ophthalmic studies that assessed hydroxychloroquine-induced retinopathy based on fundus photography and autofluorescence, visual field examination, spectral domainOCT, and mfERG. They evaluated the incidence of retinopathy, after classifying the disease in three types: parafoveal (retinal changes $2^{\circ}-6^{\circ}$ from the fovea), pericentral (retinal changes $>8^{\circ}$ from the fovea), or mixed (retinal changes in both parafoveal and pericentral areas). ${ }^{17}$ They presumed that the percentages of parafoveal, pericentral, and mixed retinal lesions were $76 \%, 12 \%$, and $12 \%$, respectively. ${ }^{17}$

The reduction of mfERG amplitudes seems to correspond to the severity of histologic lesions and morphologic alterations observed in fundus autofluorescence, but the delay in implicit times is less frequent in toxic retinopathy of hydroxychloroquine. ${ }^{18}$ This observation agrees with the findings of our study. mfERG can reveal early functional

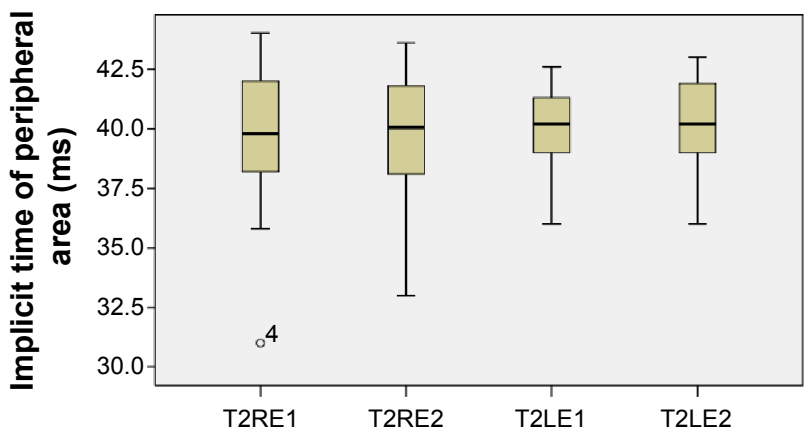

Figure 6 Implicit times of peripheral responses.

Notes: Boxplots of peripheral implicit time (ms) mean values for each eye at the first and second visits.

Abbreviations: LE, left eye; RE, right eye; T2LEI, peripheral area, left eye, first visit; T2LE2, peripheral area, left eye, second visit; T2REI, peripheral area, right eye, first visit; T2RE2, peripheral area, right eye, second visit. 
abnormalities, which are first detected in cones and the pericentral area, even in normal fundus. ${ }^{18}$ The alterations of $\mathrm{mfERG}$ responses have been correlated with cumulative doses stronger than the daily dose. ${ }^{19}$ Treatment withdrawal seems to result in improved retinal responses, affecting mainly their amplitudes, as we have also mentioned. ${ }^{20}$ Moreover, the sensitivity and specificity of mfERG appear greater compared to visual acuity and color vision testing, Amsler grid, and full-field ERG, especially in evaluating cone dysfunction. ${ }^{21,22}$ Reduced central retinal responses could be helpful in differentiating hydroxychloroquine-induced maculopathy from other retinal diseases such as age-related macular degeneration. ${ }^{22}$ However, some studies report some variability or low sensitivity in mfERG, which must be taken into account before discontinuing any treatment. ${ }^{23}$ Patient's responsiveness shows differential sensitivity according to the test they undergo. ${ }^{24}$ In contrast to mfERG, the electro-oculogram does not seem helpful in the diagnosis of hydroxychloroquine maculopathy. ${ }^{25}$ Neubauer et al ${ }^{25}$ revealed the value of the color test, given that the sensitivity and specificity of tritan color vision in patients with normal fundus or with mild disturbances of pigmentation were found to be $64 \%$ and $63 \%$, respectively (upper threshold value of $7 \%) .{ }^{25}$ The corresponding percentages in patients with bull's eye maculopathy were $75 \%$ and $94 \%$, respectively (upper threshold value of $14.8 \%) .{ }^{25}$ In addition, the P100 latency of visual evoked potentials and photostress recovery time are able to evaluate the retinal toxicity of the drug. ${ }^{26}$

Although some authors report that mfERG is a more sensitive test compared to spectral domain-OCT and autofluorescence, according to others, automated visual field testing and spectral domain-OCT are also objective methods of detecting hydroxychloroquine-induced maculopathy, appearing to possess high sensitivity and specificity. ${ }^{27}$ Cukras et $\mathrm{al}^{27}$ assessing the retinal thickness with spectral domain-OCT, observed that patients with toxic retinopathy had thinner retina $(<40 \mu \mathrm{m})$ than patients without retinopathy. In addition, the visual field mean deviation (10-2 visual field) was $11 \mathrm{~dB}$ lower in the affected group. ${ }^{27}$ The visual field mean deviation has been correlated to $\mathrm{mfERG}$ response amplitudes. ${ }^{28}$ Marmor and Melles $^{29}$ reported the presence of parafoveal ring scotomas in patients with normal spectral domain-OCT, while all patients with parafoveal lesions on spectral domain-OCT showed parafoveal focal spots of visual field loss. Another study estimated that 10-2 visual field, spectral domain-OCT, and mfERG had high-negative and low-positive predictive values, being able to exclude retinopathy when normal but not able to confirm toxicity if the condition was pathologic. ${ }^{22}$ Abnormalities in visual field that have been found in patients with hydroxychloroquine-induced retinopathy depend on the test being used, given that central defects are seen in the 24-2 test and paracentral ones in the 10-2 test. ${ }^{30}$ Frequency-doubling Humphrey perimetry and white-on-white Humphrey visual field perimetry (HVFP) can both be helpful for the diagnosis of retinal abnormalities caused by hydroxychloroquine. ${ }^{31}$ Microperimetry has detected early toxic lesions even when autofluorescence, HVFP, and mfERG appeared normal. ${ }^{32}$

Disturbances of the photoreceptors in the level of the inner and outer segment junction have been confirmed with ultrahigh-resolution OCT in symptomatic patients affected by hydroxychloroquine retinopathy. ${ }^{33}$ These abnormalities have been correlated with visual field defects and mfERG responses. ${ }^{33}$ Spectral domain-OCT changes can be identified not only in the early or late stages of toxic retinopathy but also in the absence of symptoms or fundus lesions. ${ }^{34}$ Moreover, hydroxychloroquine-induced retinopathy is characterized by thinning of peripapillary retinal nerve fiber layer, perifoveal ganglion cell layer, and inner plexiform layers, which can be independent of functional or structural clinical changes of photoreceptors or retinal pigment epithelial cell layers. ${ }^{35}$ The importance of recognizing early changes in spectral domain-OCT, before retinal pigment epithelium is affected, is highlighted by Marmor and $\mathrm{Hu},{ }^{36}$ who diagnosed progressive loss of foveal thickness and cone structure in the retina of patients receiving hydroxychloroquine, even 3 years after the cessation of the drug. In this study, we presumed that visual acuity improves along with the amplitudes of mfERG responses 6 months after the discontinuation of hydroxychloroquine therapy, detecting no differences in retinal thickness. More studies are needed to clarify whether ocular findings should influence the course of therapy. However, almost all authors agree that all patients should undergo a basic ocular examination before starting treatment to serve as a reference point and to diagnose any preexisting maculopathy. Annual screening based on fundus autofluorescence, spectral domain-OCT, and mfERG 5 years after starting hydroxychloroquine therapy is not widely recommended..$^{23,37}$

\section{Acknowledgment}

The authors alone are responsible for the contents of the paper.

\section{Disclosure}

The authors report no conflicts of interest in this work. 


\section{References}

1. Rynes RI. Antimalarial drugs in the treatment of rheumatological diseases. Br J Rheumatol. 1997;36(7):799-805.

2. Casado E, Gratacós J, Tolosa C, et al. Antimalarial myopathy: an underdiagnosed complication? Prospective longitudinal study of 119 patients. Ann Rheum Dis. 2006;65(3):385-390.

3. Stein M, Bell MJ, Ang LC. Hydroxychloroquine neuromyotoxicity. J Rheumatol. 2000;27(12):2927-2931.

4. Costedoat-Chalumeau N, Hulot JS, Amoura Z, et al. Heart conduction disorders related to antimalarials toxicity: an analysis of electrocardiograms in 85 patients treated with hydroxychloroquine for connective tissue diseases. Rheumatology (Oxford). 2007;46(5):808-810.

5. Cansu DU, Korkmaz C. Hypoglycaemia induced by hydroxychloroquine in a non-diabetic patient treated for RA. Rheumatology (Oxford). 2008;47(3):378-379

6. Makin AJ, Wendon J, Fitt S, Portmann BC, Williams R. Fulminant hepatic failure secondary to hydroxychloroquine. Gut. 1994;35(4):569-570.

7. Cohen PR. Hydroxychloroquine-associated hyperpigmentation mimicking elder abuse. Dermatol Ther (Heidelb). 2013;3(2):203-210.

8. Yam JC, Kwok AK. Ocular toxicity of hydroxychloroquine. Hong Kong Med J. 2006;12(4):294-304.

9. Geamănu Pancă A, Popa-Cherecheanu A, Marinescu B, Geamănu CD, Voinea LM. Retinal toxicity associated with chronic exposure to hydroxychloroquine and its ocular screening. J Med Life. 2014;7(3):322-326.

10. Palma Sánchez D, Rubio Velazquez E, Soro Marín S, Reyes García R. Retinal toxicity due to antimalarials: frequency and risk factors Reumatol Clin. 2013;9(5):259-262.

11. Marmor MF. New American Academy of Ophthalmology recommendations on screening for hydroxychloroquine retinopathy. Arthritis Rheum. 2003;48(6): 1764 .

12. Karkanová M, Matusková V, Vlková E, Dosková H, Uhmannová R. [The ERG contribution in early diagnosis of chloroquine and hydroxychloroquine maculopathy]. Cesk Slov Oftalmol. 2010;66(2):62-66. Czech.

13. Lai WW, Lam DS. Chloroquine-induced bull's eye maculopathy. Hong Kong Med J. 2005;11(1):55-57.

14. So SC, Hedges TR, Schuman JS, Quireza ML. Evaluation of hydroxychloroquine retinopathy with multifocal electroretinography. Ophthalmic Surg Lasers Imaging. 2003;34(3):251-258.

15. Rodríguez-Hurtado FJ, Sáez-Moreno JA, Rodríguez-Ferrer JM. Ocular toxicity and functional vision recovery in a patient treated with hydroxychloroquine. Reumatol Clin. 2015;11(3):170-173.

16. Aliferis K, Mermoud C, Safran AB. [Multifocal electroretinography in follow up of patients treated with hydroxychloroquine]. J Fr Ophtalmol. 2011;34(7):468-475. French.

17. Melles RB, Marmor MF. Pericentral retinopathy and racial differences in hydroxychloroquine toxicity. Ophthalmology. 2015;122(1):110-116.

18. Kellner U, Renner AB, Tillack H. Fundus autofluorescence and mfERG for early detection of retinal alterations in patients using chloroquine/ hydroxychloroquine. Invest Ophthalmol Vis Sci. 2006;47(8): 3531-3538.

19. Lyons JS, Severns ML. Detection of early hydroxychloroquine retinal toxicity enhanced by ring ratio analysis of multifocal electroretinography. Am J Ophthalmol. 2007;143(5):801-809.

20. Moschos MN, Moschos MM, Apostolopoulos M, Mallias JA, Bouros C, Theodossiadis GP. Assessing hydroxychloroquine toxicity by the multifocal ERG. Doc Ophthalmol. 2004;108(1):47-53.

Drug Design, Development and Therapy

\section{Publish your work in this journal}

Drug Design, Development and Therapy is an international, peerreviewed open-access journal that spans the spectrum of drug design and development through to clinical applications. Clinical outcomes, patient safety, and programs for the development and effective, safe, and sustained use of medicines are a feature of the journal, which
21. Nebbioso M, Livani ML, Steigerwalt RD, Panetta V, Rispoli E. Retina in rheumatic diseases: standard full field and multifocal electroretinography in hydroxychloroquine retinal dysfunction. Clin Exp Optom. 2011;94(3):276-283.

22. Maturi RK, Yu M, Weleber RG. Multifocal electroretinographic evaluation of long-term hydroxychloroquine users. Arch Ophthalmol. 2004; 122(7):973-981.

23. Browning DJ. Impact of the revised american academy of ophthalmology guidelines regarding hydroxychloroquine screening on actual practice. Am J Ophthalmol. 2013;155(3):418.e-428.e.

24. Marmor MF. Comparison of screening procedures in hydroxychloroquine toxicity. Arch Ophthalmol. 2012;130(4):461-469.

25. Neubauer AS, Samari-Kermani K, Schaller U, Welge-Lübetaen U, Rudolph G, Berninger T. Detecting chloroquine retinopathy: electrooculogram versus colour vision. Br J Ophthalmol. 2003;87(7): 902-908.

26. Heravian J, Saghafi M, Shoeibi N, Hassanzadeh S, Shakeri MT, Sharepoor M. A comparative study of the usefulness of color vision, photostress recovery time, and visual evoked potential tests in early detection of ocular toxicity from hydroxychloroquine. Int Ophthalmol. 2011;31(4):283-289.

27. Cukras C, Huynh N, Vitale S, Wong WT, Ferris FL 3rd, Sieving PA. Subjective and objective screening tests for hydroxychloroquine toxicity. Ophthalmology. 2015;122(2):356-366.

28. Lai TY, Ngai JW, Chan WM, Lam DS. Visual field and multifocal electroretinography and their correlations in patients on hydroxychloroquine therapy. Doc Ophthalmol. 2006;112(3):177-187.

29. Marmor MF, Melles RB. Disparity between visual fields and optical coherence tomography in hydroxychloroquine retinopathy. Ophthalmology. 2014;121(6):1257-1262.

30. Anderson C, Blaha GR, Marx JL. Humphrey visual field findings in hydroxychloroquine toxicity. Eye (Lond). 2011;25(12):1535-1545.

31. Tanga L, Centofanti M, Oddone F, et al. Retinal functional changes measured by frequency doubling technology in patients treated with hydroxychloroquine. Graefes Arch Clin Exp Ophthalmol. 2011;249(5): 715-721.

32. Jivrajka RV, Genead MA, McAnany JJ, Chow CC, Mieler WF. Microperimetric sensitivity in patients on hydroxychloroquine (Plaquenil) therapy. Eye (Lond). 2013;27(9):1044-1052.

33. Rodriguez-Padilla JA, Hedges TR 3rd, Monson B, et al. High-speed ultra-high-resolution optical coherence tomography findings in hydroxychloroquine retinopathy. Arch Ophthalmol. 2007;125(6):775-780.

34. Korah S, Kuriakose T. Optical coherence tomography in a patient with chloroquine-induced maculopathy. Indian J Ophthalmol. 2008;56(6):511-513.

35. Pasadhika S, Fishman GA. Effects of chronic exposure to hydroxychloroquine or chloroquine on inner retinal structures. Eye (Lond). 2010;24(2):340-346.

36. Marmor MF, Hu J. Effect of disease stage on progression of hydroxychloroquine retinopathy. JAMA Ophthalmol. 2014;132(9):1105-1112.

37. Marmor MF, Kellner U, Lai TY, Lyons JS, Mieler WF; American Academy of Ophthalmology. Revised recommendations on screening for chloroquine and hydroxychloroquine retinopathy. Ophthalmology. 2011;118(2):415-422.

\section{Dovepress}

has also been accepted for indexing on PubMed Central. The manuscript management system is completely online and includes a very quick and fair peer-review system, which is all easy to use. Visit http://www.dovepress.com/testimonials.php to read real quotes from published authors. 